\title{
Leukemia Inhibitory Factor Signaling Enhances Production of Galactose- Deficient IgA1 in IgA Nephropathy
}

\author{
Koshi Yamada, d Zhi qiang Huang ${ }^{a}$ Milan Raska ${ }^{a}$ e Colin Reily ${ }^{c}$ \\ Joshua C. Anderson $^{b}$ Hitoshi Suzuki ${ }^{a, d}$ Krzysztof Kiryluk $^{f} \quad$ Ali G. Gharavi $^{f}$ \\ Bruce A. Julian ${ }^{a, c}$ Christopher D. Willey ${ }^{b}$ Jan Novak ${ }^{a}$ \\ ${ }^{a}$ Department of Microbiology, University of Alabama at Birmingham, Birmingham, AL, USA; ${ }^{b}$ Department of \\ Radiation Oncology, University of Alabama at Birmingham, Birmingham, AL, USA; ' Department of Medicine, \\ University of Alabama at Birmingham, Birmingham, AL, USA; ${ }^{d}$ Department of Nephrology, Juntendo University \\ Faculty of Medicine, Tokyo, Japan; 'Department of Immunology, Palacky University Olomouc, Olomouc,

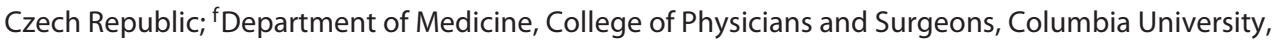 \\ New York, NY, USA
}

\section{Keywords}

Aberrant O-glycosylation · O-glycans · Autoantigen · IgA nephropathy · Leukemia inhibitory factor

\begin{abstract}
Objectives: IgA nephropathy $(\lg A N)$ is thought to involve an autoimmune process wherein galactose-deficient $\lg A 1(\mathrm{Gd}-$ $\lg \mathrm{A} 1)$, recognized as autoantigen by autoantibodies, forms pathogenic immune complexes. Mounting evidence has implicated abnormal activation of some protein-tyrosine kinases (PTKs) in IgAN. Furthermore, genome-wide association studies (GWAS) of IgAN provided insight into disease pathobiology and genetics. A GWAS locus on chromosome $22 q 12$ contains genes encoding leukemia inhibitory factor (LIF) and oncostatin M, interleukin (IL)-6-related cytokines implicated in mucosal immunity and inflammation. We have previously shown that IL-6 mediates overproduction of Gd-IgA1
\end{abstract}

K.Y. and Z.H. are co-first authors.

C.D.W. and J.N. are co-senior authors.

karger@karger.com www.karger.com/kdd

Karger"
(C) 2020 The Author(s)

Published by S. Karger AG, Basel

Karger

Open access

This article is licensed under the Creative Commons AttributionNonCommercial-NoDerivatives 4.0 International License (CC BYNC-ND) (http://www.karger.com/Services/OpenAccessLicense) Usage and distribution for commercial purposes as well as any distribution of modified material requires written permission. through aberrant STAT3 activation. Here, we show that LIF enhanced production of Gd-IgA1 in IgA1-secreting cells of patients with IgAN and provide initial analyses of LIF signaling. Methods: We characterized LIF signaling that is involved in the overproduction of Gd-IgA1, using IgA1-secreting cell lines derived from peripheral blood of patients with IgAN and healthy controls $(\mathrm{HC})$. We used global PTK activity profiling, immunoblotting, lectin ELISA, and siRNA knock-down. Results: LIF stimulation did not significantly affect production of total $\lg \mathrm{A} 1$ in $\lg \mathrm{A} 1$-secreting cells from patients with IgAN or HC. However, LIF increased production of Gd-IgA1, but only in the cells from patients with IgAN. LIF stimulation enhanced phosphorylation of STAT1 in IgA1-secreting cells from patients with IgAN to a higher degree than in the cells from HC. siRNA knock-down of STAT1 blocked LIF-mediated overproduction of Gd-IgA1. Unexpectedly, this abnormal phosphorylation of STAT1 in IgA1-secreting cells from patients with IgAN was not mediated by JAK, but rather in-
Christopher D. Willey

Department of Radiation Oncology, University of Alabama at Birmingham Hazelrig Salter Radiation Oncology Center 2232C, 1700 6th Avenue South Birmingham, AL 35233 (USA) cwilley@uabmc.edu

Jan Novak

Department of Microbiology

University of Alabama at Birmingham

845 19th Street South, BBRB 761A, Birmingham, AL 35294 (USA)

jannovak@uab.edu 
volved activation of Src-family PTKs (SFKs). Conclusion: Abnormal LIF/STAT1 signaling represents another pathway potentially leading to overproduction of Gd-lgA1 in IgAN, providing possible explanation for the phenotype associated with chromosome 22q12 GWAS locus. Abnormal LIF/ STAT1 signaling and the associated SFKs may represent potential diagnostic and/or therapeutic targets in IgAN.

(c) 2020 The Author(s)

Published by S. Karger AG, Basel

\section{Introduction}

Immunoglobulin A (IgA) nephropathy (IgAN), first described by Berger and Hinglais in 1968 [1], is the most common form of primary glomerulonephritis in the world [2]. Up to $40 \%$ of patients' progress to end-stage renal disease within 20 years of diagnosis [3], as there is no disease-specific therapy [4]. Diagnosis is based on evaluation of a renal biopsy, which shows characteristic IgA-containing immunodeposits in the glomerular mesangium with $\operatorname{IgA}$ as the predominant or co-dominant immunoglobulin. These deposits are of IgA1 isotype [5] and are enriched for IgA1 glycoforms with some $O$-glycans deficient in galactose (galactose-deficient IgA1; GdIgA1) $[6,7]$.

IgAN has been characterized as an autoimmune disease, wherein the kidneys are injured as innocent bystanders, likely due to immunodeposits derived from circulating immune complexes consisting of Gd-IgA1 (autoantigen) bound by Gd-IgA1-specific autoantibodies [4, 8, PMID: 31444275]. According to a multihit pathogenesis model $[9,10]$, the overproduction of aberrantly glycosylated IgA1 [11], the first hit, is central to the disease process.

The initial understanding of the mechanisms involved in production of Gd-IgA1 in IgAN was enabled by development of IgA1-producing cell lines derived from peripheral blood of patients with IgAN and healthy controls (HC) [12]. Analysis of the expression and activity of key $O$-glycosylation enzymes revealed important differences between IgA1-producing cells from patients with IgAN and healthy and disease controls. Specifically, expression of genes encoding core $1 \beta 1,3$-galactosyltransferase (C1GALT1) and C1GALT1-specificchaperone(COSMC) was lower and, conversely, expression of ST6GALNAC2 gene encoding ST6GalNAc-II was higher in cells from IgAN patients versus controls. Enzyme activities of C1GALT1 and ST6GalNAc-II mirrored the expression of the corresponding genes. Follow-up studies indicated that some cytokines (e.g., interleukin [IL]-4 and IL-6) can alter the expression of C1GALT1, COSMC, and/or ST6GALNAC2 genes to further dysregulate activities of the encoded enzymes [12-14]. These findings together suggested that elevated production of Gd-IgA1 in patients with IgAN was due to an abnormal biosynthesis in IgA1-secreting cells rather than galactose removal/degradation. This conclusion was further supported by 2 genome-wide association studies (GWAS) that implicated variants of C1GALT1 and COSMC in Gd-IgA1 serum levels $[15,16]$.

Genetic and genomic studies of immune endophenotypes have provided novel insights into the genetic architecture of complex traits and enhanced subclassification of several autoimmune and inflammatory disorders [17-20]. GWAS identified several IgAN susceptibility loci, providing potential clues regarding the pathways driving the pathogenesis of IgAN. The implicated pathways include the antigen processing and presentation pathways (3 loci on chromosome 6q21 in the MHC region), mucosal-immunity pathways (chromosomes 22q12, HORMAD2 locus; $8 \mathrm{q} 23$, alpha-defensin locus; and 17q13, TNFSF13 locus), and the alternative complement pathway (chromosome 1q32 complement factor $\mathrm{H}$ and CFHR locus) [21-23]. Notably, the locus on chromosome 22q12 also influences serum IgA levels. This locus is large and encompasses several candidate genes, including those encoding leukemia inhibitory factor (LIF) and oncostatin M (OSM).

LIF and OSM are members of the IL- 6 family. These cytokines are expressed in the mucosal tissues and have immunoregulatory properties $[24,25]$. Concerning mucosal tissues, upper-respiratory tract infections are often observed at the clinical disease onset and associate with exacerbated manifestations in the patients with IgAN. We have previously shown that IL-6 induces an abnormal signaling in IgA1-producing cells from patients with IgAN. This process involves enhanced and extended signaling through the JAK/STAT3 pathway, leading to elevated production of Gd-IgA1 [26]. In this study, we assessed the effect of LIF and OSM on IgA and Gd-IgA1 production by IgA1-secreting cell lines and determined the signaling mechanisms responsible for LIF-mediated enhancement of Gd-IgA1 production.

\section{Methods}

Study Design and Preparation of EBV-Immortalized

IgA1-Secreting Cells

We assessed the effect of LIF and OSM on IgA and Gd-IgA1 production and examined LIF-signaling mechanisms in IgA1-producing cells from patients with IgAN and HC. Protocols for ob- 
Table 1. Clinical characteristics of the volunteers

\begin{tabular}{|c|c|c|c|c|c|c|}
\hline & Age, years & Race $^{\mathrm{A}}$ & $\operatorname{Sex}^{B}$ & $\mathrm{SCr}, \mathrm{mg} / \mathrm{dL}$ & $\mathrm{Upr}^{\mathrm{C}}$ & Ubld $^{\mathrm{D}}$ \\
\hline $\mathrm{HC}(n=5)$ & $46.7 \pm 23.8$ & $\mathrm{~W}=5$ & $\mathrm{M}=2, \mathrm{~F}=3$ & $0.85 \pm 0.21$ & 0 & $0^{\mathrm{E}}$ \\
\hline IgAN patients $(n=5)$ & $25.2 \pm 14.9$ & $\mathrm{~W}=3, \mathrm{~A}=1, \mathrm{~B}=1$ & $\mathrm{M}=4, \mathrm{~F}=1$ & $1.25 \pm 0.23$ & $2.4 \pm 1.5^{*}$ & $1.8 \pm 1.3^{*}$ \\
\hline $\begin{array}{l}\text { Data expressed as } \\
{ }^{*} p<0.05 \\
{ }^{\text {A }} \text { Race; } \mathrm{W} \text {, white; } \\
\text { B Sex; } \mathrm{M} \text {, male; } \mathrm{F}, \mathrm{f} \\
{ }^{\mathrm{C}} \text { Urinary protein } \\
{ }^{\mathrm{D}} \text { Urinary blood wa } \\
\text { were significantly high } \\
\text { E Excludes } 1 \text { female } \\
\text { HC, healthy contro }\end{array}$ & $\begin{array}{l}\text { black. } \\
\text { d by urine te } \\
\text { ed by urine t } \\
\text { ose for HC. } \\
\text { aturia attribu } \\
\text { gA nephropa }\end{array}$ & $\begin{array}{l}\text { ips, scale }(0 \text { to }+4) \text {. } \\
\text { trips, scale }(0 \text { to }+3) \text {. A } \\
\text { to menses. }\end{array}$ & nounts of urina & protein and $\mathrm{u}$ & nary blood & IgAN patients \\
\hline
\end{tabular}

taining the blood samples for isolation of cells were approved by the Institutional Review Board for Human Use of the University of Alabama at Birmingham, and the samples were obtained after written informed consent.

Blood samples were collected by venipuncture from 5 patients with biopsy-proven IgAN and 5 HC. Mononuclear cells from blood were isolated by Ficoll-Hypaque density gradient and thereafter immortalized by infection with EBV [12].

IgA1-secreting cell lines were subcloned by limiting dilution [12]. We randomly selected IgA1-secreting cell lines from patients with $\operatorname{IgAN}(n=5)$ and $\mathrm{HC}(n=5$; Table 1$)$. Cells were grown in RPMI 1,640 supplemented with $20 \%$ fetal bovine serum, $100 \mathrm{U} / \mathrm{mL}$ of penicillin, and $0.1 \mathrm{mg} / \mathrm{mL}$ of streptomycin in a humidified $\mathrm{CO}_{2}$ $(5 \%)$ incubator at $37^{\circ} \mathrm{C}$. Cell viability was assessed by using trypan blue exclusion.

Treatment of IgA1-Secreting Cells with LIF, OSM, IL-6, and JAK2 Inhibitor

IgA1-secreting cells were plated at $1 \times 10^{5}$ cells/well in 24 -well plates, treated with LIF (40 ng/mL), OSM (40 ng/mL), and IL-6 (40 $\mathrm{ng} / \mathrm{mL}$ ) in the absence or presence of JAK2 inhibitor AZD1480 (LC Laboratories, Woburn, MA, USA; $0-0.3 \mu \mathrm{M}$ ). The cells were preincubated with the inhibitor for $1 \mathrm{~h}$ before addition of cytokine. Samples of culture medium were harvested after 5 days for analyses of total IgA1 and Gd-IgA1.

\section{Determination of Total IgA Concentration}

Total IgA was measured by ELISA. Ninety-six-well plates were coated with $0.1 \mu \mathrm{g} /$ well of goat $\operatorname{IgG} \mathrm{F}(\mathrm{ab})_{2}$ specific for human IgA (Jackson ImmunoResearch Inc., West Grove, PA, USA), blocked with $1 \%$ BSA in PBS with $0.05 \%$ Tween 20 (PBS-T), washed, and incubated with serially diluted samples of cell-culture supernatants from IgA-producing cells. Serially diluted standardized serum (Bio-Rad, Hercules, CA, USA) was used to generate a calibration curve for IgA quantification. Bound IgA was detected after addition of biotinylated goat IgG $\mathrm{F}\left(\mathrm{ab}^{\prime}\right)_{2}$ specific for human IgA (Biosource, San Diego, CA, USA), followed by avidin-horseradish peroxidase conjugate (Extravidin; Sigma, St. Louis, MO, USA) and peroxidase substrate o-phenylenediamine-hydrogen peroxide (Sigma). Optical densities were measured at $490 \mathrm{~nm}$ using an EL808 microplate reader (BioTek, Winooski, VT, USA).
As indicated above, we have cloned IgA-secreting cells and confirmed that the secreted IgA is exclusively IgA1 [12]. However, as the ELISA is not specific for IgA1 due to the use of polyclonal anti-IgA antibody, we refer to the measured analyte as IgA for formal reasons.

\section{Gd-IgA1 Assay}

Gd-IgA1 secreted in culture medium was measured by lectin ELISA. Microplates coated with $0.25 \mu \mathrm{g} /$ well of goat IgG F(ab') specific for human IgA (Jackson ImmunoResearch Inc.) were blocked with $1 \%$ BSA in PBS-T, washed, and incubated with cellculture supernatant added at $100 \mathrm{ng}$ of IgA per well and incubated overnight at $4^{\circ} \mathrm{C}$. Captured IgA was desialylated using neuraminidase (Arthrobacter ureafaciens; Glyko, Toronto, Canada) at $2 \mathrm{mU} /$ $\mathrm{mL}$ for $3 \mathrm{~h}$ at $37^{\circ} \mathrm{C}$. Gd-IgA 1 was detected using biotinylated lectin from Helix aspersa (Sigma; HAA) specific for terminal GalNAc, at $2 \mu \mathrm{g} / \mathrm{mL}$ in $1 \%$ BSA in PBS-T, followed by avidin-horseradish peroxidase conjugate (Sigma) and peroxidase substrate o-phenylenediamine-hydrogen peroxide (Sigma). Optical densities were measured at $490 \mathrm{~nm}$. The Gd-IgA1 concentration was expressed in units (U) defined as the ratio of OD determined for the individual sample and the OD for a standard Gd-IgA1 (Ale) myeloma protein. Specifically, $100 \mathrm{U}$ was defined as the OD of $100 \mathrm{ng}$ of the standard Gd-IgA1 protein [26].

\section{SDS-PAGE and Western Blot Analysis}

LIF-treated cells were pelleted by centrifugation, washed using ice-cold PBS, and lysed in M-PER lysis buffer containing protease inhibitor cocktail and phosphatase inhibitor cocktail (Thermo Fisher Scientific, Waltham, MA, USA). Cell debris was removed by centrifugation for $10 \mathrm{~min}$ at $20,000 \mathrm{~g}$ at $4{ }^{\circ} \mathrm{C}$. Protein concentrations in the supernatants were measured using a protein assay kit (Bio-Rad); aliquots corresponding to $7 \mu \mathrm{g} /$ lane of total protein were separated by SDS-PAGE and transferred to PVDF membrane for Western-blot analysis. After the transfer, the membranes were blocked using Superblock (Thermo Fisher Scientific) and incubated with phospho-Y701-STAT1-specific rabbit polyclonal antibodies, diluted 1:800 in blocking buffer, or with STAT1-specific mouse monoclonal antibodies diluted 1:10,000 (R\&D Systems, Minneapolis, MN, USA). Bound antibodies were detected by addition of HRP-conjugated anti-rabbit $(1: 4,000)$ or anti-mouse $(1: 10,000)$ 


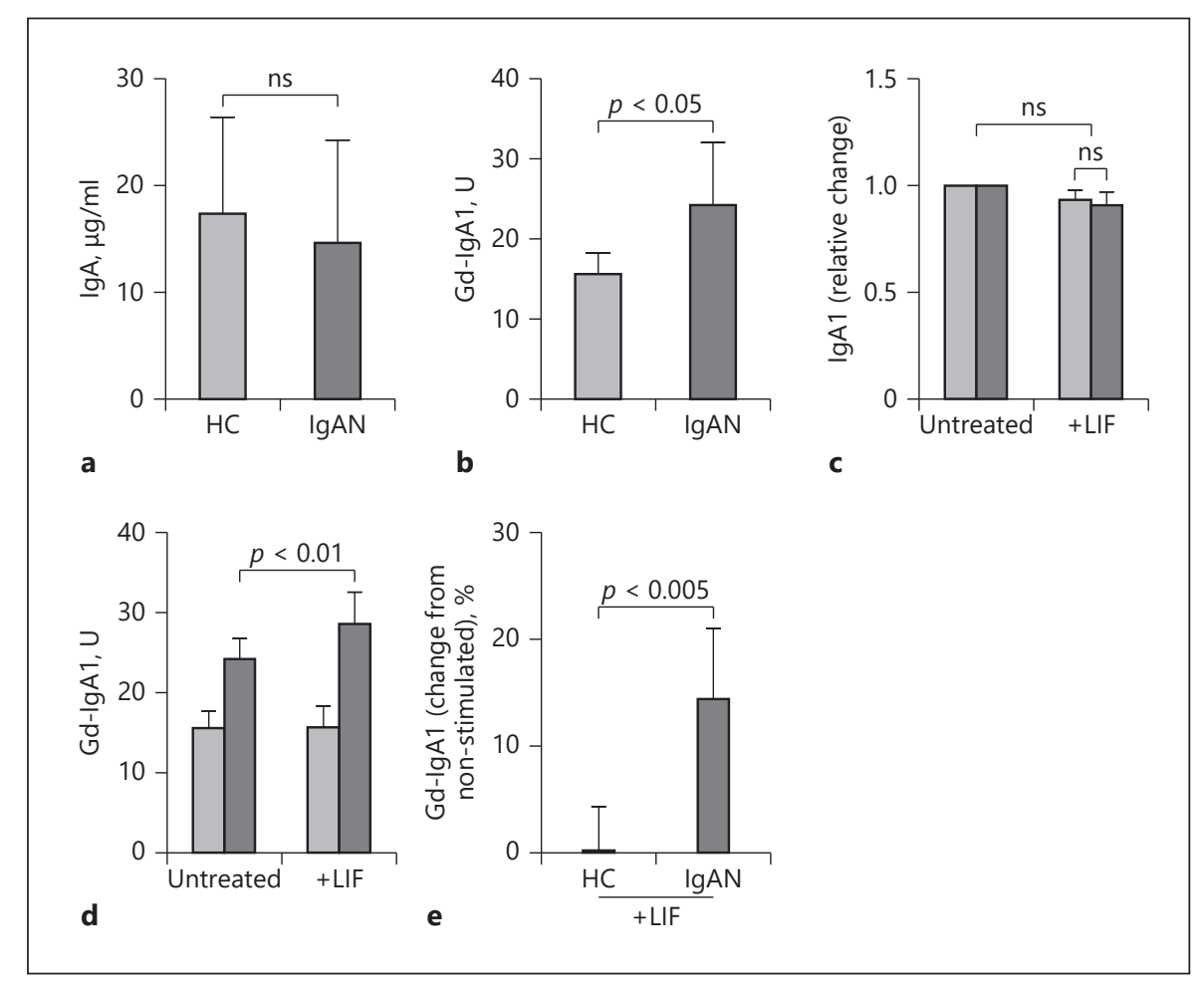

Fig. 1. IgA1 and Gd-IgA1 production by IgA1-secreting cell lines with or without LIF stimulation. IgA1-secreting cells derived from EBV-immortalized PBMCs from $5 \mathrm{HC}$ and 5 patients with IgAN were stimulated with LIF (final concentration $40 \mathrm{ng} / \mathrm{mL}$ in all experiments) or mock-stimulated (untreated). a IgA1 concentrations in cell-culture supernatants of IgA1-producing cells from $\mathrm{HC}$ and IgAN patients were comparable in the absence of LIF. $\mathbf{b}$ IgA1-producing cells from IgAN patients secreted more Gd-IgA1 than those from HC in the absence of LIF. c LIF treatment did not

IgG antibodies (Southern Biotech, Birmingham, AL, USA), respectively, followed by addition of chemiluminescence substrate (Thermo Fisher Scientific). The detected bands were visualized on a Kodak radiography film. Densitometric evaluation with ImageJ software was used with in vitro titration of STAT1 in cellular lysates for calibration of densitometric data.

\section{qRT-PCR Analysis}

RNA was isolated from $2 \times 10^{5}$ cells using RNeasy 96 Mini Kit (Qiagen, Hilden, Germany), converted to cDNA by SuperScript III First-Strand Synthesis SuperMix kit (Invitrogen, Carlsbad, CA, USA). Levels of STAT1 and GAPDH transcripts were determined by real-time RT-PCR using LightCycler 480 DNA SYBR Green I Master chemistry on LightCycler 480 instrument (Roche, Basel, Switzerland). Results were expressed as the fold change versus the result for the corresponding GAPDH housekeeping gene mRNA values using the $2^{-\Delta \Delta C T}$ method [27].

\section{STAT1 siRNA Treatment}

IgA1-producing cell lines derived from peripheral-blood mononuclear cells (PBMC) from 3 patients with IgAN (IgAN cells) and 3 significantly alter IgA1 production by IgA1-secreting cells from $\mathrm{HC}$ and IgAN patients. d, e LIF treatment increased Gd-IgA1 production in cells from IgAN patients but not in those from HC (relative change $14.4 \pm 6.6$ vs. $0.0 \pm 4.1 \%$ ). White and black bars show results for $\mathrm{HC}$ and IgAN patients, respectively. Mean values \pm SD are shown for data from 3 independent experiments. One-hundred units of Gd-IgA1 was defined as the OD of $100 \mathrm{ng}$ of the standard Gd-IgA1. Gd-IgA1, galactose-deficient IgA1; HC, healthy controls; LIF, leukemia inhibitory factor; ns, not significant.

HC cells were transfected by ON-TARGETplus SMARTpool siRNAs (Thermo Fisher Scientific) specific for human STAT1. ON-TARGETplus Non-targeting SMARTpool siRNAs served as a control. Cells were inoculated at density $5 \times 10^{5} / \mathrm{mL} 24 \mathrm{~h}$ before siRNAs were added, following our previously published protocol [26]. Before transfection, the cells were harvested by centrifugation for $10 \mathrm{~min}$ at $300 \mathrm{~g}$ and resuspended at room temperature in Nucleofector Solution C (Lonza, Basel, Switzerland) at density of $2.5 \times 10^{6}$ cells per $100 \mu \mathrm{L}$ for each transfection. After addition of $1.4 \mu \mathrm{g}$ of individual siRNA, cells were pulsed in Amaxa nucleofector II (Lonza) using program $\mathrm{X}-001$, immediately transferred to a 24 -well panel containing $1.4 \mathrm{~mL}$ of culture medium, and incubated in humidified $\mathrm{CO}_{2}$ incubator at $37^{\circ} \mathrm{C}$. Twenty-four hours after transfection, the knock-down efficiency was determined by RT-PCR. The knock-down effect was expressed as cDNA level of the individual gene normalized to GAPDH, after respective siRNA treatment, divided by respective value obtained after treatment by nontargeting siRNA.

\section{Kinomic Profiling}

Cell lysates from IgAN and HC IgA1-secreting cell lines $(n=3$ for each) were subjected to kinomic analysis using high-through- 


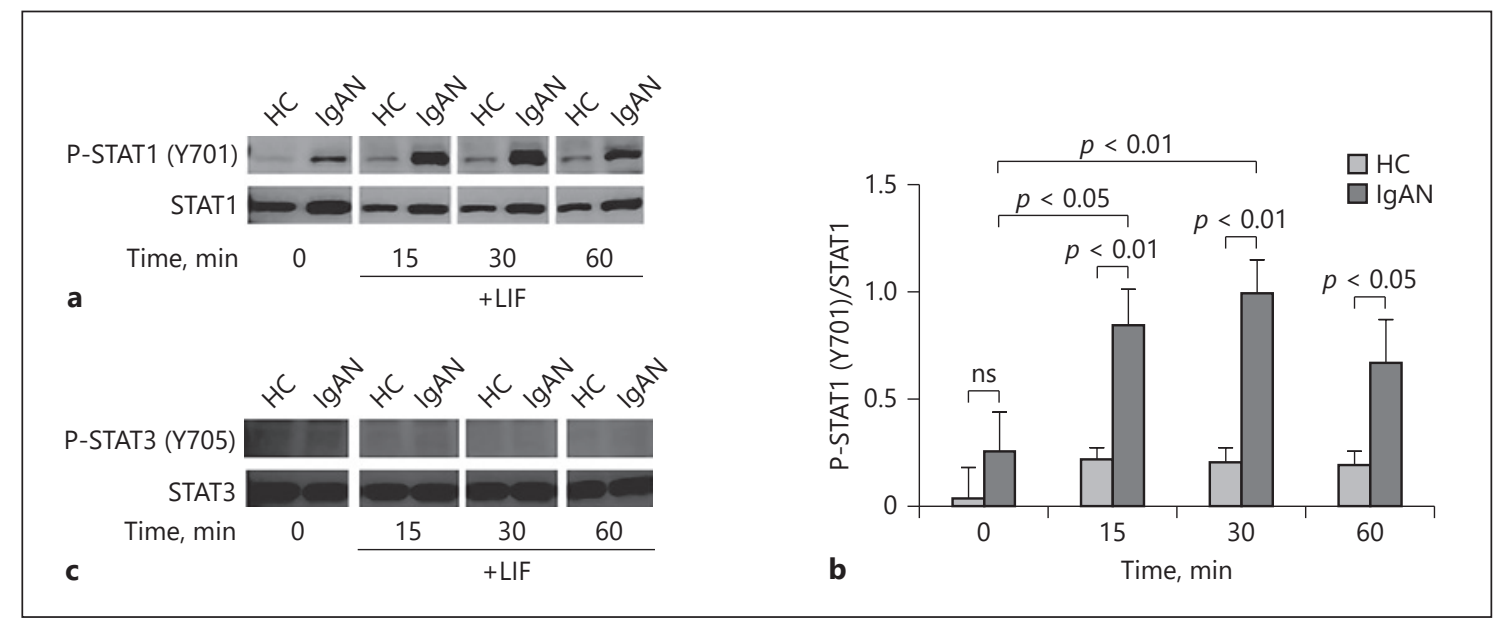

Fig. 2. LIF induced robust STAT1 phosphorylation in IgA1-secreting cell lines from IgAN patients. a Time course of LIF-induced STAT1 phosphorylation (Y701) in IgA1-secreting cell lines derived from EBV-immortalized PBMC of $\mathrm{HC}$ and IgAN patients. Total STAT1 protein served as a loading control. The experiments were repeated twice using IgA1-producing cell lines from 3 IgAN patients and $3 \mathrm{HC}$; a typical example for one of each is shown. LIF induced STAT1 phosphorylation in cells from IgAN patients to a higher degree than in cells from HC. Phosphorylation of STAT1

put phosphopeptide microarrays. All analyses were performed in triplicate. Kinomic profiling was performed using cell lysates obtained by lysing $5 \times 10^{5}$ cells with M-PER lysis buffer, as described above in the section on Western blot, and analyzed using the PamStation ${ }^{\circledR} 12$ platform (PamGene, 's-Hertogenbosch, The Netherlands) in the UAB Kinome Core laboratory (www.kinomecore. com). After protein quantification by BCA method (Thermo Fisher Scientific), lysates (12.5 $\mu$ g protein) were loaded in kinase reaction buffer onto the protein tyrosine kinase (PTK) PamChip (PamGene). This platform utilizes a high-throughput peptide microarray system analyzing 144 individual tyrosine-phosphorylatable peptides imprinted and immobilized in a 3-D format to assess kinomic activity in cell lysates. FITC-conjugated anti-phospho-tyrosine antibody (PY20, PamGene) was used for visualization. Raw images were gridded and analyzed using Evolve microarray software (PamGene). BioNavigator software (PamGene) was used for data transformation into kinetic (initial velocity) and steady state (post wash) values across multiple exposure times. Altered kinases, upstream of the altered peptides, were identified via BioNavigator UpKin PamApp (PTK version 14.0 and STK version 8.0). Briefly, this in silico tool uses kinase scoring for each peptide from phosphoNET (www.phosphonet.ca). Putative upstream kinases were mapped by analyzing results obtained on Pam Chip based off of PhosphoNET database (www.phosphonet.ca). Kinase activities were scored based on the collective changes of peptide sets between different groups. KSTAT, a kinase-associated statistic of significance in the change of phosphorylation, was scored from means of signal-to-noise ratio of peptides associated with this kinase. A larger value indicates larger change of the peptides in the same direction. Another kinase-associated statistic assessment, specificity, was calculated by a permutation test to determine the association plateaued by $30 \mathrm{~min}$ after stimulation with LIF. b Densitometric evaluation of P-STAT1 (Y701) relative to total STAT1 using IgA1secreting cells from $3 \mathrm{HC}$ and 3 IgAN patients stimulated with LIF. Mean values \pm SD from 2 similar experiments are shown. c LIF treatment did not induce phosphorylation of STAT3 (Y705) in cells from either IgAN patients or HC. HC, healthy controls; LIF, leukemia inhibitory factor; IgAN, IgA nephropathy; ns, not significant.

of the special set of peptides with the significance of a kinase. The kinases with specificity scores $>0.5$ were then uploaded to GeneGo MetaCore (portal.genego.com, Thompson Reuters, New York, NY, USA) for network analysis. Cell lysates from IgAN and HC IgA1-secreting cell lines ( $n=3$ for each group) were stimulated with LIF, $5 \mathrm{ng} / \mathrm{mL}$ for $20 \mathrm{~min}$, prior to kinomic analysis. Mockstimulated cells served as negative controls.

\section{Statistical Analyses}

Results were expressed as mean \pm SD. Data were analyzed using 2 -tailed Student $t$ test (unpaired or paired, as applicable). For analyzing multiple groups, 1-way ANOVA was used, and values of $p<0.05$ were regarded significant.

\section{Results}

\section{Participants in Research}

The demographic characteristics and clinical laboratory findings for 5 patients with IgAN and $5 \mathrm{HC}$ are listed in Table 1.

\section{LIF Increased Production of Gd-IgA1 in}

IgA1-Producing Cell Lines from IgAN Patients

EBV-immortalized IgA1-producing cell lines derived from PBMC of IgAN patients (IgAN) and HC secreted comparable amounts of IgA1 into the culture medium under baseline conditions (Fig. 1a). IgA1-producing cells 


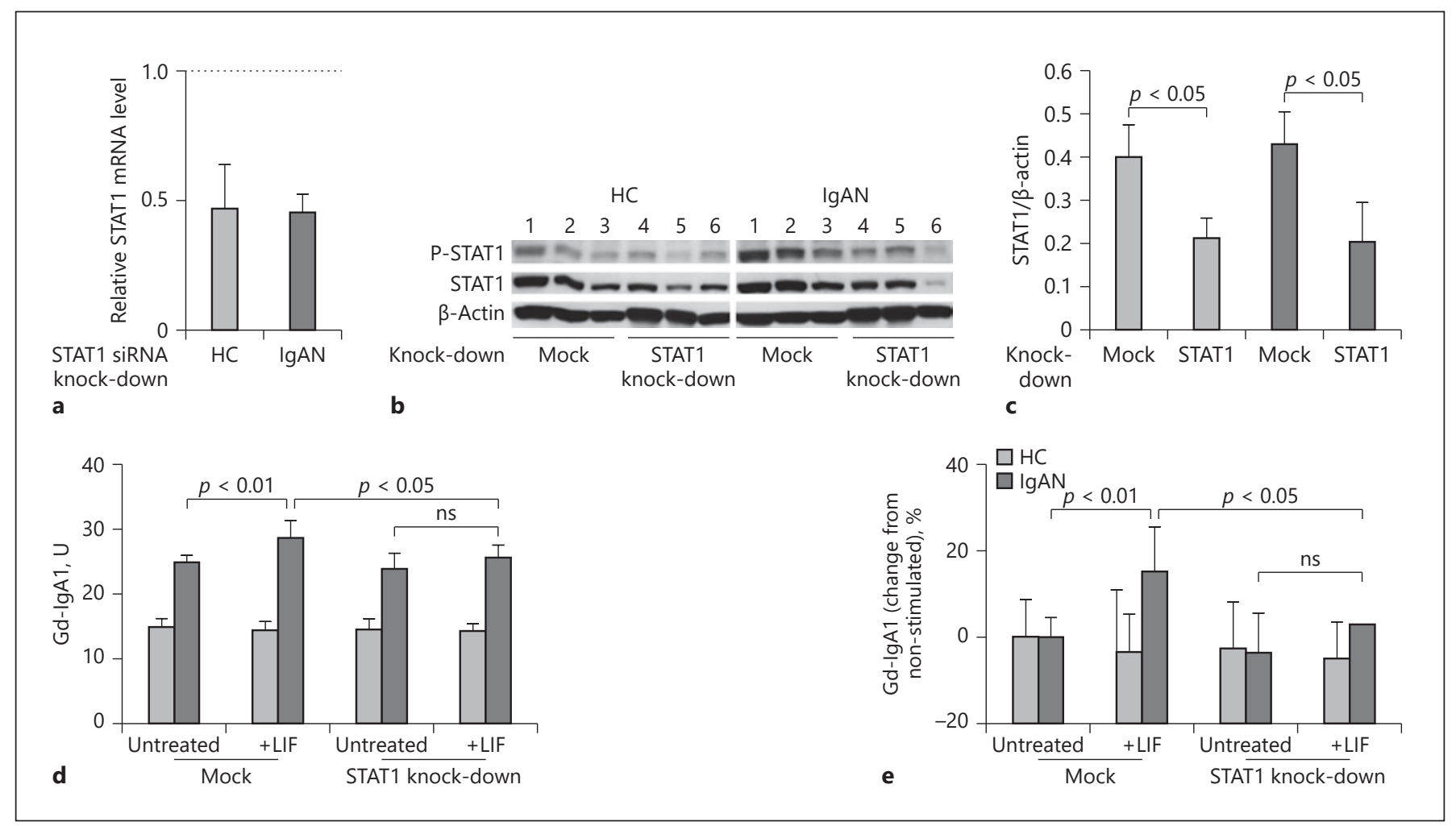

Fig. 3. siRNA knock-down confirmed that STAT1 mediated overproduction of Gd-IgA1 induced by LIF in IgA1-secreting cells from IgAN patients. a Real-Time RT-PCR analysis of STAT1 transcripts confirmed robust siRNA knock-down in IgA1-secreting cells from $\mathrm{HC}$ and IgAN patients (IgAN). Bars represent mean values (+SD) of STAT1 mRNA levels after gene-specific knockdown in IgA1-secreting cell lines derived from PBMC of $3 \mathrm{HC}$ and 3 IgAN patients. STAT1 transcript level in mock-control samples (transfection with non-targeting siRNA) was set to 1 for each cell type (i.e., HC, IgAN). b Reduction in P-STAT1 and STAT1 protein amount relative to $\beta$-actin after siRNA knock-down was con-

from IgAN patients secreted more Gd-IgA1 than cells from HCs (Fig. 1b). LIF treatment did not significantly alter total IgA1 production (Fig. 1c). However, the cells from IgAN patients secreted more Gd-IgAl after LIF stimulation in comparison to HC cells (Fig. 1d, e).

We also assessed OSM for its potential effect on IgA or Gd-IgA1 production. OSM did not significantly alter total IgA1 production or production of Gd-IgA1 (data not shown) and, thus, we focused the follow-up studies on LIF.

\section{LIF Treatment Enhanced STAT1 Phosphorylation in} IgA1-Secreting Cells from IgAN Patients to a Greater Extent than in Cells from $\mathrm{HC}$

LIF induced STAT1 phosphorylation at Y701 in IgA1producing cell lines (Fig. 2a). STAT1 phosphorylation at firmed by Western blotting. c Densitometric analysis of STAT1 protein relative to $\beta$-actin after siRNA knock-down for cells from $3 \mathrm{HC}$ and 3 IgAN patients. d, e LIF-induced overproduction of Gd-IgA1 in IgAN cells was blocked by STAT1 siRNA knockdown. Mean values \pm SD are from 3 samples in each group and expressed in $U$ (d) or as a relative change from non-stimulated cells (e). One-hundred units of Gd-IgA1 was defined as the OD of $100 \mathrm{ng}$ of the standard Gd-IgA1. HC, healthy controls; LIF, leukemia inhibitory factor; Gd-IgA1, galactose-deficient IgA1; IgAN, IgA nephropathy; ns, not significant.

Y701 plateaued after 30 min of LIF stimulation and was significantly higher in IgAN cells than in HC cells (Fig. 2b). Next, we tested whether STAT3, used for signaling by some IL-6-family cytokines, may also be involved in LIF signaling. LIF stimulation of the IgA1-producing cells from IgAN patients or HC did not activate STAT3 (Fig. 2c). Furthermore, OSM stimulation did not significantly alter STAT1 phosphorylation at Y701 in any IgA1producing cell line (data not shown).

\section{STAT1 siRNA Knock-Down Reduced LIF-Mediated Increase in Gd-IgA1 Production}

To confirm that STAT1 mediated the LIF-induced GdIgA1 overproduction in IgA1-producing cell lines from IgAN patients, we used siRNA knock-down technique. 


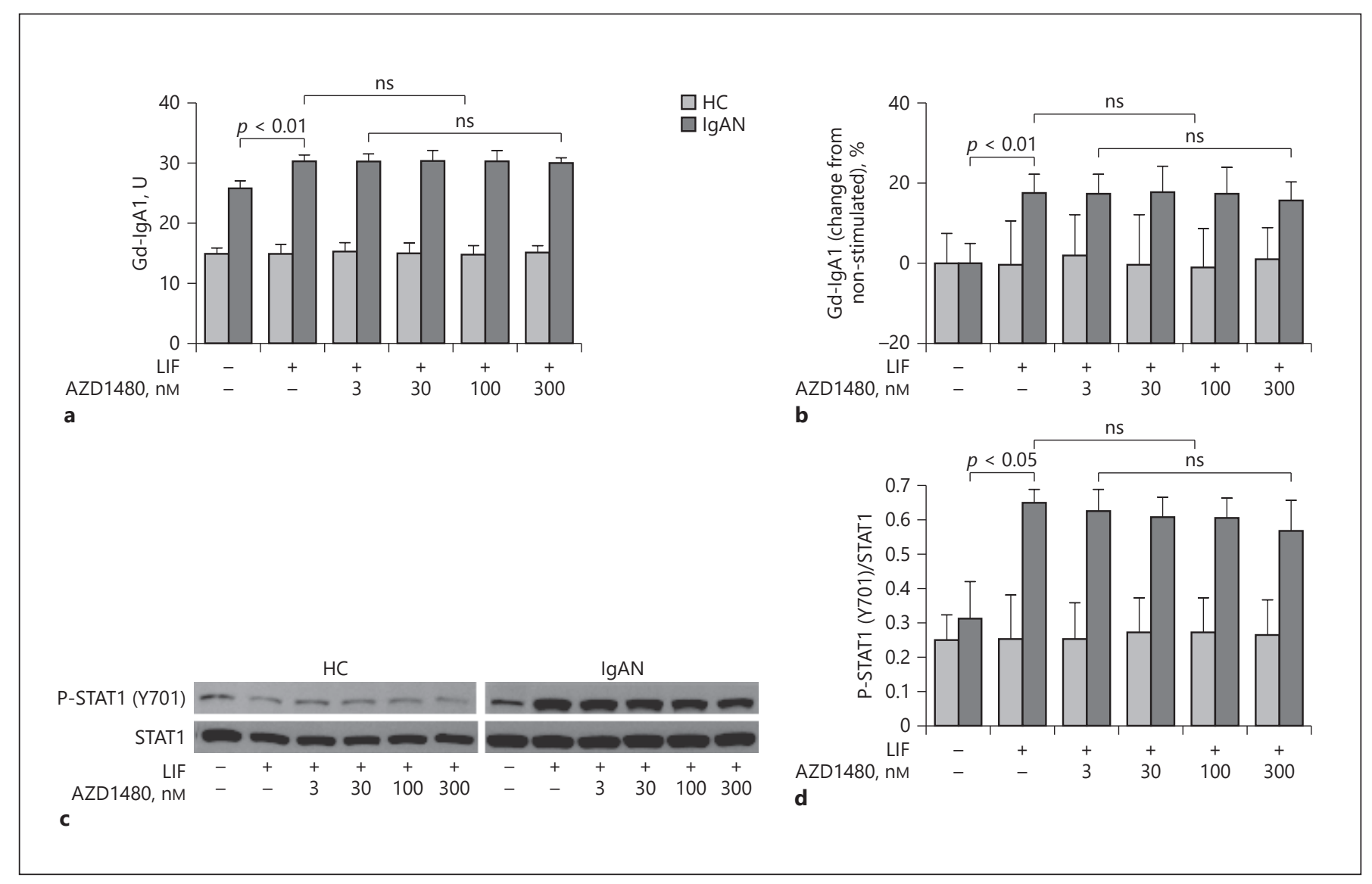

Fig. 4. AZD1480, an inhibitor of JAK2, did not inhibit LIF-mediated increase in production of Gd-IgA1 or phosphorylation of STAT1. To assess whether LIF signaling in IgA1-producing cells was mediated by JAK2, we used a JAK2 inhibitor, AZD1480, with and without LIF stimulation. a, b AZD1,480 did not inhibit LIFmediated increase of Gd-IgA1. Mean values \pm SD are from 3 samples in each group and expressed in $U$ (a) or as a relative change

siRNA knock-down reduced mRNA levels of STAT1 in cells from IgAN patients as well as HC by approximately $50 \%$ (Fig. 3a). Corresponding reduction of STAT1 protein $(>50 \%)$ and its phosphorylated form were confirmed by immunodetection (Fig. 3b, c). Analysis of secreted IgA1 and Gd-IgA1 revealed that STAT1 siRNA knock-down significantly reduced the LIF-induced overproduction of Gd-IgA1 (Fig. 3d, e). However, STAT1 knock-down did not reduce baseline production of Gd-IgA1 by IgAN cells to the level observed for HC cells (Fig. 3d, e).

Increased Production of Gd-IgA1 Induced by LIF Was Not Affected by a JAK2 Inhibitor

To test if JAK2 pathway was involved in LIF-mediated overproduction of Gd-IgA1, a JAK2 inhibitor, AZD1480, from non-stimulated cells (b). c, d Consistent with these findings, AZD1,480 even at high concentrations only marginally inhibited LIF-induced phosphorylation of STAT1. One-hundred units of Gd-IgA1 was defined as the OD of $100 \mathrm{ng}$ of the standard Gd-IgA1. $\mathrm{HC}$, healthy controls; LIF, leukemia inhibitory factor; Gd-IgA1, galactose-deficient IgA1; IgAN, IgA nephropathy; ns, not significant.

was used. AZD1480 did not block the LIF-enhanced production of Gd-IgA1 in IgA1-producing cell lines from IgAN patients (Fig. 4a, b). Moreover, the enhanced phosphorylation of STAT1 induced by LIF was not significantly reduced by AZD1480 (Fig. 4c, d). These results together indicated that LIF does not utilize JAK2-STAT3 pathway for signal transduction.

\section{Profiling of PTK Activities Indicated that LIF Utilized}

Different Pathways in Cells from IgAN Patients versus

HC

Kinomic studies revealed that baseline activities of several PTKs in IgA1-producing cells from IgAN patients were higher than those in cells from $\mathrm{HC}$ (Table 2a). Most of the 20 kinases with highest KSTAT and speci- 
Table 2. PTK activities in cells from IgAN patients and HC

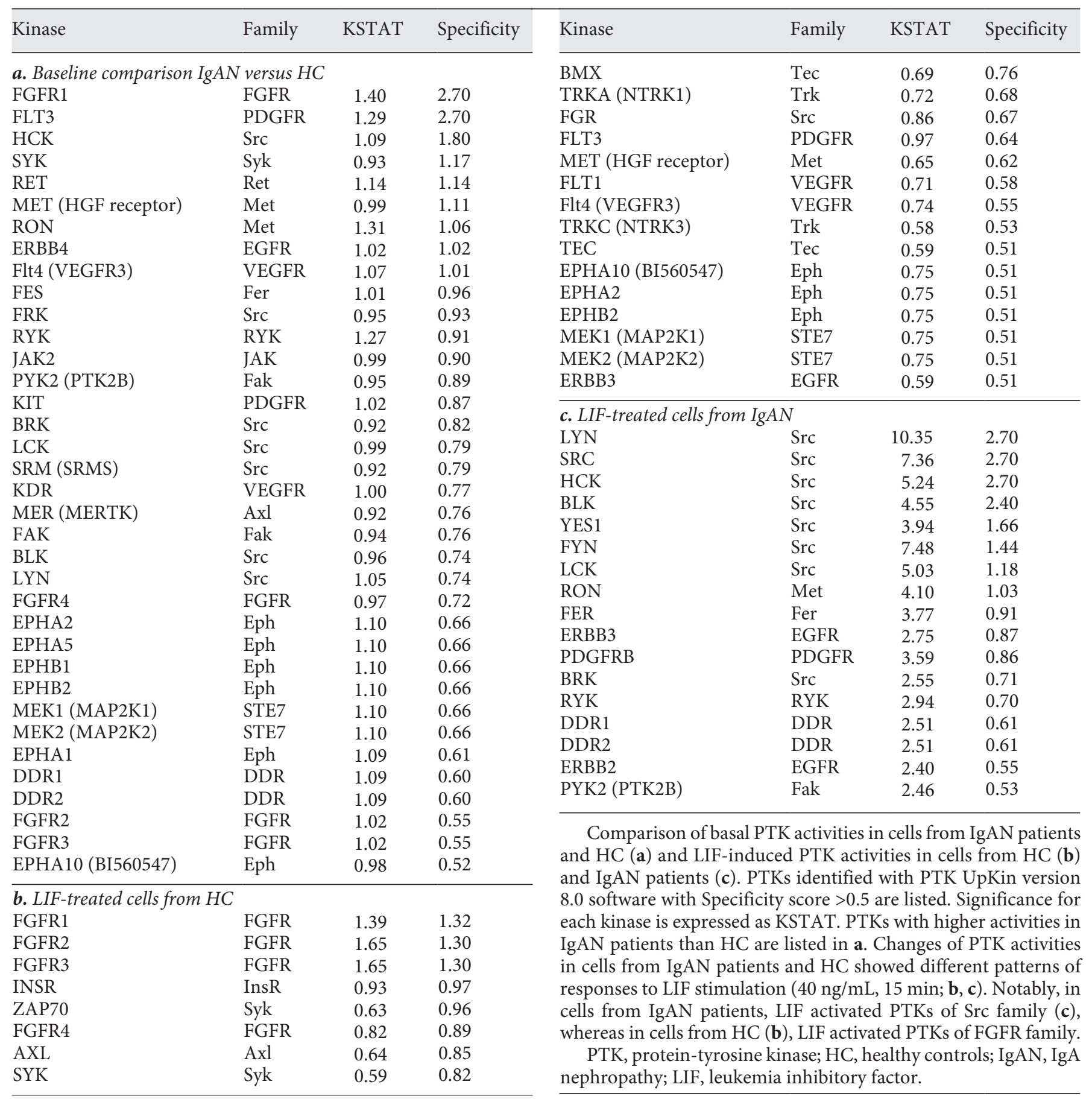

ficities belonged to families of growth factors, Eph and Src (Table 2a), the signaling pathways related to cell growth, transcription factors, and cross-activation of PTK receptors. Notably, LIF increased the activities of
PTKs in the Src family in IgA1-producing cells from IgAN patients (Table 2c) but not in cells from HC (Table 2b). Data from Table 2 were analyzed using GeneGo knowledge-base software to visualize the implicated sig- 


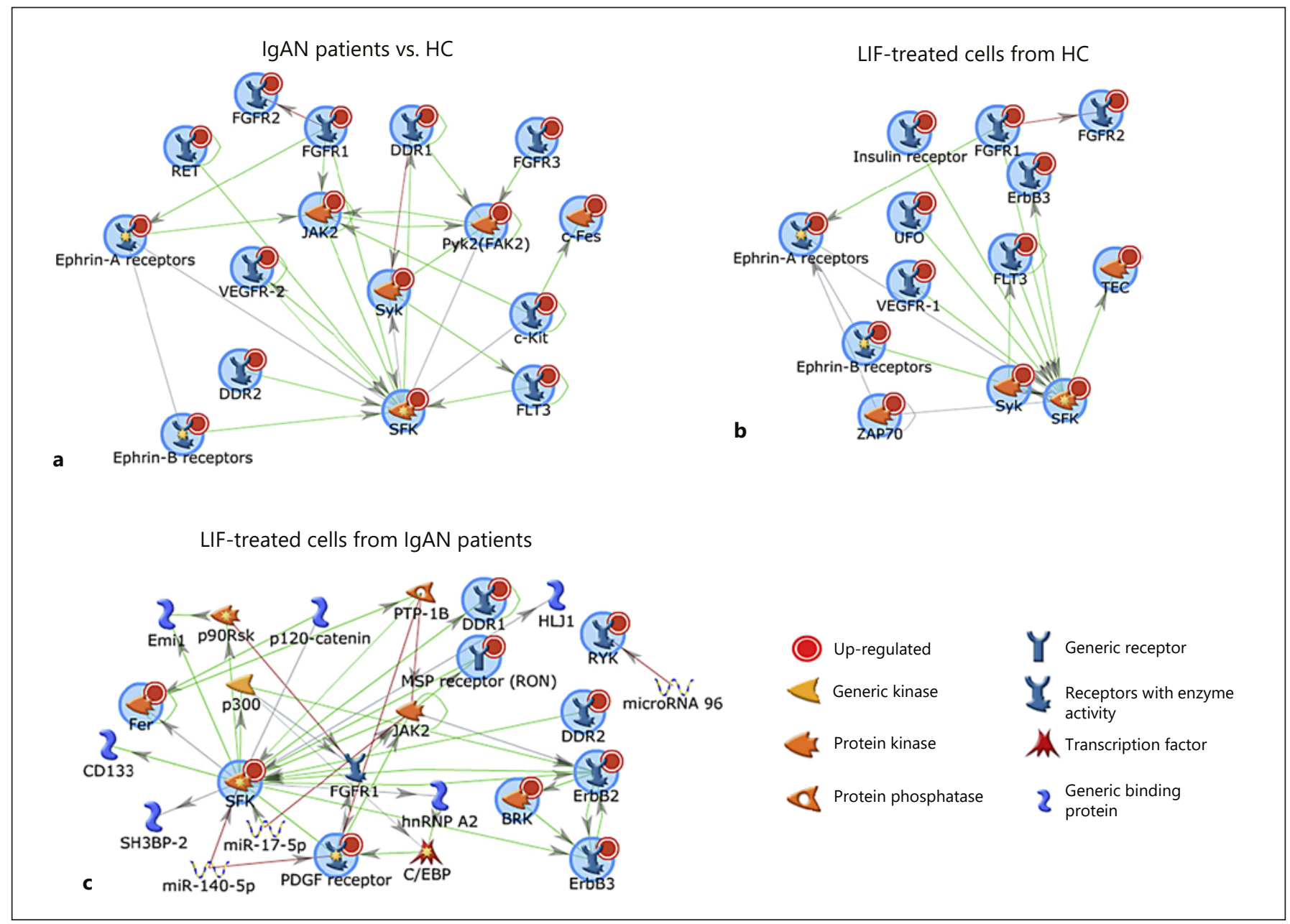

Fig. 5. PTK signaling pathways in IgA1-producing cell lines from IgAN patients (IgAN) and HC. Baseline PTK activities in cells from IgAN patients versus $\mathrm{HC}(\mathbf{a})$ and signaling pathways induced by LIF in cells from HC and IgAN patients, respectively (b, c). PTKs identified with PTK UpKin version 8.0 software in Table 2 with Specificity scores $>0.5$ were uploaded to GeneGo MetaCore using Uniprot ID and mapped using an auto-expand network algorithm $($ nMax $=50)$, with canonical pathways deselected, compounds hidden, and orphan nodes excluded. Kinases in large blue circles were input nodes. Red circles in the upper right section of a node indicate an increase in the condition (IgAN in $\mathbf{a}$; LIF in $\mathbf{b}$,

naling pathways (Fig. 5). Baseline differences between IgA1-producing cells from IgAN patients and $\mathrm{HC}$ included activities of several growth-factor-related PTKs that were elevated in the cells from IgAN patients (Fig. 5a). Moreover, LIF activated FGFR, PDGFR and EGFR systems in cells from HC (Fig. 5b), whereas Srcfamily PTKs (SFKs) were involved in the cells from IgAN patients (Fig. 5c). c). Arrowheads denote direction of literature-annotated interactions with color of line indicating type (red-negative, green-positive, gray-complex/unknown). a Activities of growth factor-associated PTKs, such as FGF and VEGF, and Eph and Syk were higher in cells from IgAN patients than in cells from HC. b LIF stimulation significantly up-regulated FGFR, PDGFR and EGFR systems in cells from HC. c LIF stimulation of cells from IgAN patients induced up-regulation of growth factors of SFKs. Symbols used in panels (a-c) are explained in the legend (bottom right). HC, healthy controls; LIF, leukemia inhibitory factor; IgAN, IgA nephropathy.

\section{Discussion}

A multihit pathogenesis model of IgAN $[9,28]$ postulates that production of aberrantly glycosylated IgA1, that is, Gd-IgA1, plays an essential role in the development and progression of the disease. Multiple lines of evidence suggest that Gd-IgA1 circulatory levels are not only increased in IgAN patients compared to HC [11] but that 
these elevated levels predict disease progression [29-31]. In addition, glomerular IgA immunodeposits in patients with IgAN are enriched for Gd-IgA1 glycoforms $[6,7]$, further supporting the role of abnormal IgA $1 O$-glycosylation in pathogenesis of IgAN $[9,10,28,32]$.

Previous studies of the origin of Gd-IgA1 indicated abnormalities in the biosynthetic process in IgA1-producing cells, specifically the altered expression of genes encoding several key glycosyltransferases [12-14]. Recent GWAS findings showed an association of IgAN with SNPs for critical glycosyltransferase and chaperone genes, C1GALT1 and COSMC. These data were validated with in vitro studies that revealed that 2 genome-wide significant loci, on chromosomes 7p21.3 and Xq24, influence GdIgA1 production $[15,16]$. Furthermore, environmental factors, such as mucosal infections, may further accentuate the aberrant $\mathrm{O}$-glycosylation of IgA1. These environmental effects are likely mediated by cytokines, such as IL-6 [13], providing a plausible connection between mucosal inflammation and kidney damage in IgAN patients.

Genetic and genomic studies have revealed gene-mediated influences in IgAN prevalence [15, 21-23, 33, 34]. Advances in genomic studies of immune endophenotypes have provided novel insights into the genetic architecture of complex traits of several autoimmune and inflammatory disorders, including inflammatory bowel disease (IBD) [35-38]. Multiple genetic-susceptibility factors for IgAN have been identified in GWAS. These GWAS loci encode genes involved in the maintenance of the intestinal epithelial barrier and/or response to mucosal pathogens, including antigen processing and presentation (3 loci in the $M H C$ region), the complement system (CFHR1/3 and ITGAM-ITGAX loci), regulation of mucosal IgA production (TNFSF13 and HORMAD/LIF/OSM loci), and innate-immunity factors (alpha-defensin, CARD9, ITGAMITGAM, and VAV3 loci). Notably, GWAS loci for IgAN are often shared with other autoimmune and inflammatory disorders, such as IBD [33]. Consistent with this finding is the observation that IgAN often complicates Crohn's disease and ulcerative colitis [39].

Other factors impacting IgA-producing cells include TNFSF13, a proliferation-inducing ligand (APRIL), and $B$-cell activating factor (BAFF) that can engage specific TNF receptors: BAFF-R (BR3), transmembrane activator and calcium modulator and cyclophilin ligand interactor, and B-cell maturation antigen. BAFF is essential for survival and maturation of B cells, sustained germinal center reaction, and support of class-switch recombination [40]. Survival of plasma cells requires APRIL and/or BAFF signaling through B-cell maturation antigen, whereas im- munoglobulin class-switched memory B cells are maintained independent of BAFF or APRIL [41]. Interestingly, transgenic mice with human BAFF exhibit an IgAN-like nephritis with IgA glomerular deposits, but only in the presence of intestinal mucosal bacteria $[42,43]$. Dysregulation of BAFF is associated with autoimmune diseases, such as systemic lupus erythematosus [44], and, thus, targeting soluble BAFF using Belimumab has been proposed for treatment of patients with lupus nephritis [45].

GWAS-identified IgAN susceptibility HORMAD/LIF/ $O S M$ locus is also a risk factor for IBD. However, the IgAN risk allele at this locus is protective against Crohn's disease $[35,46,47]$ and is associated with elevated serum IgA levels [22]. LIF and OSM are 2 cytokines from the IL-6 family involved in mucosal immunity $[22,33]$. Although the specific factors that elicit mucosal immune dysregulation remain elusive, these findings have stimulated interest in new treatment approaches, such as Budesonide (a non-systemic corticosteroid that suppresses local gut inflammation), Bortezomib (proteasome inhibitor), Blisibimob (BAFF inhibitor), and Atacicept (APRIL and BAFF inhibitor).

IL-6, the prototype of IL-6 family of cytokines, is involved in the pathogenesis of several autoimmune and inflammatory diseases [48]. IL-6 canonical signaling utilizes the JAK/STAT3 pathway [49-51], a critical interface between autoimmunity and immune deficiency. Recently, we determined that abnormal IL-6-induced signaling through IL-6 receptor-gp130 and JAK2/STAT3 pathway was associated with elevated synthesis of Gd-IgA1 by IgA1-producing cells from patients with IgAN but not HC [26]. Loss of STAT3 signaling disrupts the mucosal barrier in disease such as Crohn's disease, resulting in epithelial expression of the IL-6-like cytokine Upd3, leading to activation of JAK/STAT signaling, differentiation of cells that form the progenitor cells niche, and abnormal proliferation of progenitor cells [52]. It would be of interest to determine if similar abnormalities in progenitor cells in intestinal mucosa may be involved in IgAN. Activation of STAT transcriptional regulators by cytokine and growth-factor receptors is typically fast but transient, due to specific negative-feedback mechanisms. Abnormal activation, however, is due to unbalanced signaling or altered levels of specific JAK/STAT proteins or their negative regulators. The latter are protein inhibitors of activated STATs, protein tyrosine phosphatases, or suppressors of cytokine signaling. Abnormalities of these negative regulators are often found in pathological conditions, such as cancer and disorders of the immune systems $[53,54]$. 
In this study, we assessed differential signaling mechanisms responsible for LIF-mediated increase in GdIgA1 production using IgA 1 -secreting cell lines derived from peripheral-blood cells of patients with IgAN and HC. LIF induced STAT1 phosphorylation (Y701) to a greater degree in IgA1-producing cells from IgAN patients when compared with those from HC. STAT3 activation was not involved. LIF-induced increase in GdIgA1 production by IgA1-producing cells from IgAN patients was significantly reduced after siRNA STAT1 knock-down. Thus, abnormal STAT1 signaling induced by LIF mediated the overproduction of Gd-IgA1. Notably, STAT1 and STAT3 activation are reciprocally regulated and perturbation in their balanced expression or phosphorylation levels may re-direct cytokine/growth factor signals from proliferative to apoptotic, or from inflammatory to anti-inflammatory [55]. Signal transduction via gp130 (encoded by IL6ST gene) is mediated by the JAK/STAT pathway and can include phosphorylation of STAT1 and STAT3, as well as activation of RAS/ MAPK pathways [56]. A mutation in IL6ST is associated with loss of IL-6- and OSM-mediated signaling in hyperIgE syndrome [57].

Notably, this abnormal phosphorylation of STAT1 in IgA1-secreting cells from patients with IgAN was not mediated by JAK2. Although JAK/STAT pathway can be activated by many different cytokines, including all members of the IL-6 family [58], our data implicated a shift toward SFKs, notably Lyn, Src, Hck and Blk, and EGFR family kinases following LIF stimulation (Table 2 ). The SFKs are nonreceptor kinases, involved in multiple signaling processes that regulate key cellular functions, including proliferation, migration, differentiation, and survival. SFKs can be activated through several growth-factor or cytokine receptors, as well as through integrin activation and B- and T-cell receptor signaling. A prototypical growth factor receptor cascade involves EGF signaling through EGFR to activate Src $[59,60]$. However, Src can be activated through integrin-driven adhesion, a process that has already been linked with STAT1 activation during monocyte to macrophage maturation [61]. A more recent report implicates both EGFR and integrin pathway with STAT1 activation in monocytes that further supports the potential for non-traditional activation of STAT1 [62]. As the abnormal phosphorylation of STAT1 in IgA1-secreting cells from patients with IgAN was not mediated by JAK2, it is possible that LIF activation involved SFKs and EGFR in IgA1-producing cells from IgAN patients [63-65]. Moreover, the SFKs play multiple roles in sig- nal transduction by the B-cell antigen receptor and contribute to signaling by B-cell antigen receptor surrogates during B-lymphocyte development and maturation. Paradoxically, loss of function of SFKs results not only in the expected defects in B-cell development but also enhances onset of autoimmunity. Thus, SFKs, particularly Lyn, play both activating and inhibitory roles in B-cell function $[60,66]$. LIF-STAT1 activation enhanced activities of SFKs in Gd-IgA1-secreting cells from patients with IgAN, underscoring the pathogenic potential of these cells in IgAN.

In summary, abnormal LIF/STAT1 signaling leads to overproduction of Gd-IgA1 in IgAN, possibly explaining the phenotype associated with chromosome $22 \mathrm{q} 12$ GWAS locus. This signaling and the associated SFKs may represent diagnostic or therapeutic targets in IgAN. The emerging picture of the abnormal signaling pathways in IgA1-secreting cells from patients with IgAN induced by several cytokines of IL- 6 family requires further clarification through combined genomic, kinomic, and functional studies.

\section{Acknowledgments}

The authors thank Ms. Courtany Grammer for her assistance with collection of demographic and laboratory data.

\section{Statement of Ethics}

Protocols for obtaining the blood samples for isolation of cells were approved by the Institutional Review Board for Human Use of the University of Alabama at Birmingham, and the samples were obtained after written informed consent.

\section{Disclosure Statement}

The authors report no conflict of interest. In the interest of full disclosure, we report that B.A.J. and J.N. are co-founders and coowners of Reliant Glycosciences, LLC, while C.D.W. and J.C.A. are co-founders of Spacer Science, LLC. C.D.W. is a part-time consultant for LifeNet Health.

\section{Funding Sources}

This study has been supported in part by grants DK078244, DK082753, DK106341, and DK105124 from the National Institutes of Health and a gift from the IgAN Foundation of America. None of the funding sources had any role in the design or execution of the study, analysis of the data, or preparation of the manuscript. 


\section{Author Contributions}

K.Y. and Z.H. contributed equally to the execution of the study and writing of the first draft of the manuscript. K.K., A.G.G., B.A.J., C.D.W., and J.N. conceived the study and outlined the manuscript.
K.Y., Z.H., M.R., C.R., J.C.A., and H.S. designed, performed, and interpreted specific experiments. All authors reviewed and edited the manuscript. C.D.W. and J.N. are co-senior and co-corresponding authors.

\section{References}

1 Berger J, Hinglais N. [Intercapillary deposits of IgA-IgG]. J Urol Nephrol (Paris). 1968 Sep; 74(9):694-5.

2 Julian BA, Waldo FB, Rifai A, Mestecky J. IgA nephropathy, the most common glomerulonephritis worldwide. A neglected disease in the United States? Am J Med. 1988 Jan;84(1): $129-32$.

3 D'Amico G. Natural history of idiopathic IgA nephropathy: role of clinical and histological prognostic factors. Am J Kidney Dis. 2000 Aug;36(2):227-37.

4 Wyatt RJ, Julian BA. IgA nephropathy. N Engl J Med. 2013 Jun;368(25):2402-14.

5 Conley ME, Cooper MD, Michael AF. Selective deposition of immunoglobulin A1 in immunoglobulin A nephropathy, anaphylactoid purpura nephritis, and systemic lupus erythematosus. J Clin Invest. 1980 Dec;66(6):1432-6.

6 Allen AC, Bailey EM, Brenchley PE, Buck KS, Barratt J, Feehally J. Mesangial IgA1 in IgA nephropathy exhibits aberrant O-glycosylation: observations in three patients. Kidney Int. 2001 Sep;60(3):969-73.

7 Hiki Y, Odani H, Takahashi M, Yasuda Y, Nishimoto A, Iwase H, et al. Mass spectrometry proves under-O-glycosylation of glomerular IgA1 in IgA nephropathy. Kidney Int. 2001 Mar;59(3):1077-85.

8 Suzuki H, Fan R, Zhang Z, Brown R, Hall S, Julian BA, et al. Aberrantly glycosylated IgA1 in IgA nephropathy patients is recognized by IgG antibodies with restricted heterogeneity. J Clin Invest. 2009 Jun;119(6):1668-77.

9 Suzuki H, Kiryluk K, Novak J, Moldoveanu Z, Herr AB, Renfrow MB, et al. The pathophysiology of IgA nephropathy. J Am Soc Nephrol. 2011 Oct;22(10):1795-803.

10 Novak J, Julian BA, Mestecky J, Renfrow MB. Glycosylation of IgA1 and pathogenesis of IgA nephropathy. Semin Immunopathol. 2012 May;34(3):365-82.

11 Moldoveanu Z, Wyatt RJ, Lee JY, Tomana M, Julian BA, Mestecky J, et al. Patients with IgA nephropathy have increased serum galactosedeficient IgA1 levels. Kidney Int. 2007 Jun; 71(11):1148-54.

12 Suzuki H, Moldoveanu Z, Hall S, Brown R, Vu HL, Novak L, et al. IgA1-secreting cell lines from patients with IgA nephropathy produce aberrantly glycosylated IgA1. J Clin Invest. 2008 Feb;118(2):629-39.

13 Suzuki H, Raska M, Yamada K, Moldoveanu Z, Julian BA, Wyatt RJ, et al. Cytokines alter IgA1 O-glycosylation by dysregulating C1GalT1 and ST6GalNAc-II enzymes. J Biol Chem. 2014 Feb;289(8):5330-9.
14 Stuchlova Horynova M, Vrablikova A, Stewart TJ, Takahashi K, Czernekova L, Yamada $\mathrm{K}$, et al. $\mathrm{N}$-acetylgalactosaminide a2,6sialyltransferase II is a candidate enzyme for sialylation of galactose-deficient IgA1, the key autoantigen in IgA nephropathy. Nephrol Dial Transplant. 2015 Feb;30(2):234-8.

15 Kiryluk K, Li Y, Moldoveanu Z, Suzuki H, Reily C, Hou P, et al. GWAS for serum galactose-deficient IgA1 implicates critical genes of the O-glycosylation pathway. PLoS Genet. $2017 \mathrm{Feb} ; 13(2)$ :e1006609.

16 Gale DP, Molyneux K, Wimbury D, Higgins P, Levine AP, Caplin B, et al. Galactosylation of IgA1 is associated with common variation in C1GALT1. J Am Soc Nephrol. 2017 Jul; 28(7):2158-66.

17 Lyons PA, Rayner TF, Trivedi S, Holle JU, Watts RA, Jayne DR, et al. Genetically distinct subsets within ANCA-associated vasculitis. N Engl J Med. 2012 Jul;367(3):214-23.

18 Liang L, Willis-Owen SA, Laprise C, Wong KC, Davies GA, Hudson TJ, et al. An epigenome-wide association study of total serum immunoglobulin E concentration. Nature. 2015 Apr;520(7549):670-4.

19 Weidinger S, Gieger C, Rodriguez E, Baurecht $\mathrm{H}$, Mempel M, Klopp N, et al. Genome-wide scan on total serum IgE levels identifies FCER1A as novel susceptibility locus. PLoS Genet. 2008 Aug;4(8):e1000166.

20 Lauc G, Huffman JE, Pučić M, Zgaga L, Adamczyk B, Mužinić A, et al. Loci associated with N-glycosylation of human immunoglobulin $\mathrm{G}$ show pleiotropy with autoimmune diseases and haematological cancers. PLoS Genet. 2013;9(1):e1003225.

21 Feehally J, Farrall M, Boland A, Gale DP, Gut I, Heath S, et al. HLA has strongest association with IgA nephropathy in genome-wide analysis. J Am Soc Nephrol. 2010 Oct;21(10):17917.

22 Gharavi AG, Kiryluk K, Choi M, Li Y, Hou P, Xie J, et al. Genome-wide association study identifies susceptibility loci for IgA nephropathy. Nat Genet. 2011 Mar;43(4):321-7.

$23 \mathrm{Yu} \mathrm{XQ}$, Li M, Zhang H, Low HQ, Wei X, Wang JQ, et al. A genome-wide association study in Han Chinese identifies multiple susceptibility loci for IgA nephropathy. Nat Genet. 2011 Dec;44(2):178-82.

24 Rockman SP, Demmler K, Roczo N, Cosgriff A, Phillips WA, Thomas RJ, et al. Expression of interleukin-6, leukemia inhibitory factor and their receptors by colonic epithelium and pericryptal fibroblasts. J Gastroenterol Hepatol. 2001 Sep;16(9):991-1000.
25 Guimbaud R, Abitbol V, Bertrand V, Quartier G, Chauvelot-Moachon L, Giroud J, et al. Leukemia inhibitory factor involvement in human ulcerative colitis and its potential role in malignant course. Eur Cytokine Netw. 1998 Dec;9(4):607-12.

26 Yamada K, Huang ZQ, Raska M, Reily C, Anderson JC, Suzuki H, et al. Inhibition of STAT3 signaling reduces IgA1 autoantigen production in IgA nephropathy. Kidney Int Rep. 2017 Jul;2(6):1194-207.

27 Livak KJ, Schmittgen TD. Analysis of relative gene expression data using real-time quantitative PCR and the $2(-\Delta \Delta \mathrm{C}(\mathrm{T}))$ Method. Methods. 2001 Dec;25(4):402-8.

28 Novak J, Rizk D, Takahashi K, Zhang X, Bian $\mathrm{Q}$, Ueda $\mathrm{H}$, et al. New insights into the pathogenesis of IgA nephropathy. Kidney Dis. 2015 May;1(1):8-18.

29 Zhao N, Hou P, Lv J, Moldoveanu Z, Li Y, Kiryluk K, et al. The level of galactose-deficient IgA1 in the sera of patients with IgA nephropathy is associated with disease progression. Kidney Int. 2012 Oct;82(7):790-6.

30 Maixnerova D, Ling C, Hall S, Reily C, Brown R, Neprasova M, et al. Galactose-deficient IgA1 and the corresponding IgG autoantibodies predict IgA nephropathy progression. PLoS One. 2019 Feb;14(2):e0212254.

31 Suzuki H, Allegri L, Suzuki Y, Hall S, Moldoveanu Z, Wyatt RJ, et al. Galactose-deficient IgA1 as a candidate urinary polypeptide marker of IgA nephropathy? Dis Markers. 2016;2016:7806438.

32 Novak J, Barratt J, Julian BA, Renfrow MB. Aberrant glycosylation of the IgA1 molecule in IgA nephropathy. Semin Nephrol. 2018 Sep;38(5):461-76.

33 Kiryluk K, Li Y, Scolari F, Sanna-Cherchi S, Choi M, Verbitsky M, et al. Discovery of new risk loci for IgA nephropathy implicates genes involved in immunity against intestinal pathogens. Nat Genet. 2014 Nov;46(11): 1187-96.

34 Kiryluk K, Li Y, Sanna-Cherchi S, Rohanizadegan M, Suzuki H, Eitner F, et al. Geographic differences in genetic susceptibility to IgA nephropathy: GWAS replication study and geospatial risk analysis. PLoS Genet. 2012; 8(6):e1002765.

35 Jostins L, Ripke S, Weersma RK, Duerr RH, McGovern DP, Hui KY, et al.; International IBD Genetics Consortium (IIBDGC). Hostmicrobe interactions have shaped the genetic architecture of inflammatory bowel disease. Nature. 2012 Nov;491(7422):11924. 
36 McGovern DP, Kugathasan S, Cho JH. Genetics of inflammatory bowel diseases. Gastroenterology. 2015 Oct;149(5):1163-1176.e2.

37 Lee JC, Biasci D, Roberts R, Gearry RB, Mansfield JC, Ahmad T, et al.; UK IBD Genetics Consortium. Genome-wide association study identifies distinct genetic contributions to prognosis and susceptibility in Crohn's disease. Nat Genet. 2017 Feb;49(2):262-8.

38 de Lange KM, Moutsianas L, Lee JC, Lamb CA, Luo Y, Kennedy NA, et al. Genome-wide association study implicates immune activation of multiple integrin genes in inflammatory bowel disease. Nat Genet. 2017 Feb; 49(2):256-61.

39 Ambruzs JM, Walker PD, Larsen CP. The histopathologic spectrum of kidney biopsies in patients with inflammatory bowel disease. Clin J Am Soc Nephrol. 2014 Feb;9(2):26570.

40 Vincent FB, Saulep-Easton D, Figgett WA, Fairfax KA, Mackay F. The BAFF/APRIL system: emerging functions beyond $\mathrm{B}$ cell biology and autoimmunity. Cytokine Growth Factor Rev. 2013 Jun;24(3):203-15.

41 Avery DT, Kalled SL, Ellyard JI, Ambrose C, Bixler SA, Thien M, et al. BAFF selectively enhances the survival of plasmablasts generated from human memory B cells. J Clin Invest. 2003 Jul;112(2):286-97.

42 McCarthy DD, Chiu S, Gao Y, Summers-deLuca LE, Gommerman JL. BAFF induces a hyper-IgA syndrome in the intestinal lamina propria concomitant with IgA deposition in the kidney independent of LIGHT. Cell Immunol. 2006 Jun;241(2):85-94.

43 McCarthy DD, Kujawa J, Wilson C, Papandile A, Poreci U, Porfilio EA, et al. Mice overexpressing BAFF develop a commensal floradependent, IgA-associated nephropathy. J Clin Invest. 2011 Oct;121(10):3991-4002.

44 Vincent FB, Northcott M, Hoi A, Mackay F, Morand EF. Association of serum B cell activating factor from the tumour necrosis factor family (BAFF) and a proliferation-inducing ligand (APRIL) with central nervous system and renal disease in systemic lupus erythematosus. Lupus. 2013 Aug;22(9):873-84.

45 Dooley MA, Houssiau F, Aranow C, D’Cruz DP, Askanase A, Roth DA, et al.; BLISS-52 and -76 Study Groups. Effect of belimumab treatment on renal outcomes: results from the phase 3 belimumab clinical trials in patients with SLE. Lupus. 2013 Jan;22(1):63-72.
46 Franke A, McGovern DP, Barrett JC, Wang K, Radford-Smith GL, Ahmad T, et al. Genomewide meta-analysis increases to 71 the number of confirmed Crohn's disease susceptibility loci. Nat Genet. 2010 Dec;42(12):1118-25.

47 Imielinski M, Baldassano RN, Griffiths A Russell RK, Annese V, Dubinsky M, et al.; Western Regional Alliance for Pediatric IBD; International IBD Genetics Consortium; NIDDK IBD Genetics Consortium; BelgianFrench IBD Consortium; Wellcome Trust Case Control Consortium. Common variants at five new loci associated with early-onset inflammatory bowel disease. Nat Genet. 2009 Dec;41(12):1335-40.

48 Papanicolaou DA, Wilder RL, Manolagas SC, Chrousos GP. The pathophysiologic roles of interleukin-6 in human disease. Ann Intern Med. 1998 Jan;128(2):127-37.

49 Bende RJ, Jochems GJ, Frame TH, Klein MR, van Eijk RV, van Lier RA, et al. Effects of IL-4, IL-5, and IL- 6 on growth and immunoglobulin production of Epstein-Barr virus-infected human B cells. Cell Immunol. 1992 Sep; 143(2):310-23.

50 Lin L, Benson DM Jr, DeAngelis S, Bakan CE, Li PK, Li C, et al. A small molecule, LLL12 inhibits constitutive STAT3 and IL-6-induced STAT3 signaling and exhibits potent growth suppressive activity in human multiple myeloma cells. Int J Cancer. 2012 Mar;130(6): 1459-69.

51 Lue C, Kiyono H, McGhee JR, Fujihashi K, Kishimoto T, Hirano T, et al. Recombinant human interleukin 6 (rhIL-6) promotes the terminal differentiation of in vivo-activated human B cells into antibody-secreting cells. Cell Immunol. $1991 \mathrm{Feb}$;132(2):423-32.

52 Zhang L, Turner B, Ribbeck K, Ten Hagen KG. Loss of the mucosal barrier alters the progenitor cell niche via Janus kinase/signal transducer and activator of transcription (JAK/STAT) signaling. J Biol Chem. 2017 Dec;292(52):21231-42.

53 Aaronson DS, Horvath CM. A road map for those who don't know JAK-STAT. Science. 2002 May;296(5573):1653-5.

54 Shuai K, Liu B. Regulation of JAK-STAT signalling in the immune system. Nat Rev Immunol. 2003 Nov;3(11):900-11.

55 Avalle L, Pensa S, Regis G, Novelli F, Poli V. STAT1 and STAT3 in tumorigenesis: A matter of balance. JAK-STAT. 2012 Apr;1(2):6572 .
56 O'Shea JJ, Plenge R. JAK and STAT signaling molecules in immunoregulation and immune-mediated disease. Immunity. 2012 Apr;36(4):542-50.

57 Schwerd T, Twigg SR, Aschenbrenner D, Manrique S, Miller KA, Taylor IB, et al. A biallelic mutation in IL6ST encoding the GP130 co-receptor causes immunodeficiency and craniosynostosis. J Exp Med. 2017 Sep;214(9): 2547-62.

58 Aparicio-Siegmund S, Sommer J, Monhasery N, Schwanbeck R, Keil E, Finkenstädt D, et al. Inhibition of protein kinase II (CK2) prevents induced signal transducer and activator of transcription (STAT) $1 / 3$ and constitutive STAT3 activation. Oncotarget. 2014 Apr; 5(8):2131-48.

59 Espada J, Martín-Pérez J. An update on Src family of nonreceptor tyrosine kinases biology. Int Rev Cell Mol Biol. 2017;331:83-122.

60 Parsons SJ, Parsons JT. Src family kinases, key regulators of signal transduction. Oncogene. 2004 Oct;23(48):7906-9.

61 Coccia EM, Del Russo N, Stellacci E, Testa U, Marziali G, Battistini A. STAT1 activation during monocyte to macrophage maturation: role of adhesion molecules. Int Immunol. 1999 Jul;11(7):1075-83.

62 Collins-McMillen D, Stevenson EV, Kim JH, Lee BJ, Cieply SJ, Nogalski MT, et al. Human cytomegalovirus utilizes a nontraditional signal transducer and activator of transcription 1 activation cascade via signaling through epidermal growth factor receptor and integrins to efficiently promote the motility, differentiation, and polarization of infected monocytes. J Virol. 2017 Nov;91(24):91.

63 Ernst M, Gearing DP, Dunn AR. Functional and biochemical association of Hck with the LIF/IL-6 receptor signal transducing subunit gp130 in embryonic stem cells. EMBO J. 1994 Apr;13(7):1574-84.

64 Fang Y, Zhong L, Lin M, Zhou X, Jing H, Ying $M$, et al. MEK/ERK dependent activation of STAT1 mediates dasatinib-induced differentiation of acute myeloid leukemia. PLoS One. 2013 Jun;8(6):e66915.

65 Bhattacharjee A, Pal S, Feldman GM, Cathcart MK. Hck is a key regulator of gene expression in alternatively activated human monocytes. J Biol Chem. 2011 Oct;286(42): 36709-23.

66 Gauld SB, Cambier JC. Src-family kinases in B-cell development and signaling. Oncogene. 2004 Oct;23(48):8001-6. 